[60] - Zur Normierbarlieit eines algemeinen topologischen linearen Raumes, Studia Math. 5 (1934), pp. 29-33.

[61] K. Kuratowski, Topologie I, and II, Mon. Mat. 20 and 21, Warszawa 1958 and 1961

[62] J. Lindenstrauss, On non-linear projections in Banach spaces, Michigan Math. Journ. 11 (1964) pp. 268-287.

[63] D. Maharam, On homogeneous measure algebras, Proc. Nat. Acad. Sci. USA 28 (1942), pp. 108-111.

[64] B. M. Makaro , On the moment problem in certain functional spaces (Russian), Dokl. Akad. Nauk. SSSR (N. S.) 127 (1959), pp. 957, 960.

[65] S. Mazur, Une remarque sur l'homéomorphie des champs fonctionels, Studia Math. 1 (1929), pp. 83-85.

[66] - and. W. Orlicz, Sur les espaces métriques linéaires, I, Studia Math. 10 (1948), pp. 184-208; II, ibidem 13 (1953), pp. 137-179.

[67] E. Michael, Convex structure and continuous selection, Canad. Journ. Math. 11 (1959), pp. 556-576.

[68] B. S. Miatiagin, Approximative dimension and bases in nuclear spaces, Usp. Mat. Nauk. 16, no. 4 (1961), pp. 63-132.

[69] A. Pełczyński, On the isomorphism of spaces mand M, Bull. Acad. Polon. Sei., Sér. sci. math. astr. et phys., 7 (1958). pp. 695-696.

[70] - Projections in certain Banach spaces, Studia Math. 19 (1960), pp. 209-228.

[71] - On the degree of condensation of compact spaces and its revationship with the spaces of continuous functions, Fund. Math. (to appear).

[72] - Problems P364-P369, Colloq. Math. 9 (1962), pp. 168-169.

[73] - On the approximation of F-spaces by finite-dimensional spaces, Bull. Acad. Polon. Sci., Cl. III, 5 (1957), pp. 879-881.

[74] - and Z. Semadeni, Spaces of continuous functions (III), Studia Math. 18 (1959), pp. 211-222.

[75] S. Rolewicz, On spaces of holomorphic functions, Studia Math. 21 (1961). pp. $135-160$.

[76] - On Cauchy-Hadanard formula for Köthe power spaces, Bull. Acad. Polon. Sci., Sér, sci. math. astr. et phys., 10 (1962), pp. 211-216.

[77] V. Ju. Sandberg, On Lipschitz spaces (Russian), Dok. Akad. Nauk SSSR (N. S.) 145 (1962), pp. 284-286.

[78] I. J. Schoenberg, Metric spaces and positive dejinite functions, Trans. Amer. Math. Soc. 44 (1938), pp. 522-536.

[79] - Metric spuces and completely monotone functions, Ann. of Math. 39, no. 4 (1938), pp. 811-841.

[80] I. Singer, Problema omeomorfismului spatilor Banach separabile inifinit-dimensionale, Acad. R. P. Romine, An. Romino-Soviet Ser. mat. fiz. 12 (1958), no. 4 (27), pp. $5 \cdot 24$.

[81] M. H. Stone, Notes on integration II, Proc. Nat. Acad. Sci. USA. 34 (1948), pp. 447-455.

[82] C. Bessaga and V. Klee, Two topological properties of topological linear spaces, Israel J. Math. 2 (1964).

Reģu par la Rédaction le 24. 2. 1964.

\section{On Egoroff's theorem}

by

E. P. Rozycki (Buffalo, N. Y.)

I. Although Egoroff's theorem [6] is usually stated for sequences one finds it used in certain instances when the collection of functions inrolved is non-denumerable ([5], [7]). However, several counter-examples exist in the literature which show that the conclusion of the theorem does not in general follow in this case ([2], [8], [9], [10]). Hahn and Rosenthal [3] must have realized this, although no reference to a counterexample is mentioned, since they state and prove a non-denumerable analogue to Egoroff's theorem, but by placing certain restrictions on the functions not found in the original form of the theorem. Essentially, they prove:

Let $m$ be a measure function on an additive class of sets $A$ of a space $X, A$ an element of $\boldsymbol{A}$ such that $m(A)<+\infty$ and $F$ a real function defined on $A \times(0,1)$ such that for each $x \in A, F(x, \cdot)$ is continuous on $(0,1)$ and for each $t \in(0,1), F(\cdot, t)$ is measurable on $A$.. If

$$
\lim _{t \rightarrow 0} F^{\prime}(x, t)=G(x)
$$

a.e. on $A$, where $G$ is finite a.e. on $A$, then, for each $\eta>0$, there exists a set $B \subset A$ such that $m(A-B)<\eta$ and the convergence of $F(\cdot, t)$ to $G$ is uniform on $B$.

It is the purpose of this note to weaken the hypotheses of the above theorem. In what follows $F, m, A, G$ and $\boldsymbol{A}$ are to have the same significance as above as well as the notation $F(x, \cdot)$ and $F^{\prime}(\cdot, t)$. We obtain our results by replacing the set $(0,1)$ with an infinite set $M$ and varying its nature.

II. We first suppose that $M$ is an infinite subset of a topological space $Y$ which is Hausdorff and second countable while its elosure, el $M$, is countably compact (see Hall and Spencer [4]). This allows us to assume without any loss that if we let $M^{\prime}$ denote the derived set of $M$ and $H$ a countable subset of $M$ dense in $M$, then, if $p \in \mathrm{cl} M$ but $p \in H$, then $p \in M^{\prime}-M r$. Let 1.s.c. (u.s.c.) denote lower [upper] semi-continuous. If $f$ is a real function defined on a set $E$ and $H \subset E$ then the 
symbol $\sup f(H)$ means the supremum of the set $\{f(y) \mid y \in H\}$. A corresponding meaning is given to $\inf f(H)$. The main result of this section is the following theorem.

THEOREM 1. If (i) $F(\cdot, t)$ is measurable on $A$ for each $t \in M$, (ii) $F(x, \cdot\rangle$ is 1.s.c. [u.s.c.] on $M$ for each $x \in A$, (iii) $\lim F(x, t)$ exists and equals $G(x)$ a.e. on $A$, and (iv) $G(x) \leqslant F(x, t)[G(x) \geqslant F(x, t)]$ for each $x \in A$ and for every $t$ in some neighborhood $V$ of $a \in M^{\prime}$, then for each prescribed $\eta>0$ there exists some set $B C A$ with $m(A-B)<\eta$ on which $\lim F(x, t)$ $=G(x)$ uniformly.

The theorem cited in the first section turns out to be a corollary of the above theorem. In order to prove the above theorem we need the following lemma.

Lemcua. If $F(x, \cdot)$ is l.s.c. [u.s.c.] on $M$ for each $x \in A$ and $F(\cdot, t)$ is measurable on $A$ for every $t \in M$ and if

$$
\begin{aligned}
\sup F(\cdot, M)(x) & =\sup F(x, M), \\
\inf F(\cdot, M)(x) & =\inf F^{\prime}(x, M), \\
\limsup _{t \rightarrow a} F^{\prime}(\cdot, t)(x) & =\limsup _{t \rightarrow a} F^{\prime}(x, t), \\
\liminf _{t \rightarrow a}(\cdot, t)(x) & =\liminf _{t \rightarrow a} F^{\prime}(x, t),
\end{aligned}
$$

then both $\sup F(\cdot, M)[\inf F(\cdot, M)]$ and $\limsup _{t \rightarrow a} \mathrm{~F}(\cdot, t)\left[\liminf _{t \rightarrow a} F(\cdot, t)\right]$ are measurable on $A$, the latter holding for all $a \in M^{\prime}$.

We prove only the l.s.c. part of the lemma, the proof of the u.s.c. part being similar.

Since $M$ is second countable, then it is separable. Let $H=\left\{r_{n}\right\} \subset M$ be a countable set dense in $M$. Place $f_{n}(x)=F\left(x, r_{n}\right)$ and $C(x)=\sup f_{n}(x)$.

We assert that $C(x)=\sup F^{\prime}(x, M)$. Indeed, all we need show is $C(x)$ $\geqslant \sup F(x, M)$ since $C(x) \leqslant \sup F^{\prime}(x, M)$ follows from $H \subset M$. Therefore, suppose to the contrary that for some $x^{*} \in A, C\left(x^{*}\right)<\sup F^{*}\left(x^{*}, M\right)$. Then there exists a $t^{*} \in M$ such that

Hence

$$
C\left(x^{*}\right)<E\left(x^{*}, t^{*}\right) \leqslant \sup F\left(x^{*}, M\right) .
$$

$$
f_{n}\left(x^{*}\right)=F^{\prime}\left(x^{*}, r_{n}\right) \leqslant C\left(x^{*}\right)<F^{\prime}\left(x^{*}, t^{*}\right) \leqslant \sup F\left(x^{*}, M\right) .
$$

Since this is true for every $n$, then $t^{*} \frac{\dot{\epsilon}}{H}$ and so $t^{*} \in M^{\prime}$. From this folIows $t^{*} \in H^{\prime}$. Therefore, if we let $N\left(t^{*}\right)$ denote the family of all neighborhoods of $t^{*}$, then $U \cap H \neq \emptyset$ for every $U \in N\left(t^{*}\right)$; thus

$$
\inf F^{\prime}\left(x^{*}, U\right) \leqslant C\left(x^{*}\right)<F^{\prime}\left(x^{*}, t^{*}\right)
$$

But then

$$
\sup \left\{\inf F^{\prime}\left(x^{*}, U\right), U \in N\left(t_{*}\right)\right\} \leqslant C\left(x^{*}\right)<F^{\prime}\left(x^{*}, t^{*}\right)
$$

which contradicts the hypothesis that $F\left(x^{*}, \cdot\right)$ is 1.s.c. on $M$. Thus our assertion holds and since $C$ is measurable on $A$, so is $\sup F(\cdot, M I)$.

To show the second part of the lemma we make use of a countable basis $\left\{U_{n}\right\}$ at $a$ such that $U_{n+1} \subset U_{n}$. Define the sequence of functions $\left\{F_{n}\right\}$ by $F_{n}(x)=\sup F\left(x, U_{n}\right)$. Then by what has just preceded, $F_{n}$ is measurable on $A$ for every $n$. Since $\left\{U_{n}\right\}$ is a decreasing sequence of sets, $\left\{F_{n}\right\}$ is a decreasing sequence of functions and thus $\lim F_{n}(x)$ exists for ${ }^{2}$ each $x \in A$, say $D(x)$. It follows immediately that $\limsup _{t \rightarrow a} F(x, t) \leqslant D(x)$. The opposite inequality also follows. To see this, let $\stackrel{t \rightarrow a}{V}$ be an arbitrary neighborhood of $a$. Then there is an integer $N$ such that $U_{N} \subset V$. This implies $\sup F\left(x, U_{N}\right) \leqslant \sup F(x, V)$. But then $D(x) \leqslant \sup F(x, T)$ and the desired result follows from the arbitrariness of $V$. Thus $D(x)=\limsup F(x, t)$ Now $D$ is measurable on $\mathcal{A}$; consequently so is $\limsup _{t \rightarrow a} F(\cdot, t)$.

As above we now give a proof of the 1.s.c. part of Theorem 1, the proof of the u.s.c. part being similar. By removing from $A$ a set of measure zero we may assume that $F(\cdot, t)$ converges to $G$ everywhere on $A$ and that $G$ is finite on $A$. Let $\left\{U_{n}\right\}$ and $\left\{F_{n}\right\}$ have the same significance as in the second part of the proof of the Lemma. According to this lemma we know each $F_{n}$ as well as $\limsup _{t \rightarrow a} F(\cdot, t)$ is measurable on $A$. Since

$$
\limsup _{t \rightarrow a} F^{\prime}(x, t)=\lim _{t \rightarrow a} F^{\prime}(x, t)=G(x)=\lim _{n} F_{n}(x),
$$

the same must be true of $G$. Also, in virtue of the fact that $G$ is finite on $A$ and the properties $F_{n}(x) \geqslant F_{n+1}(x), x \in A$, and (iv) (Theorem 1), for each $\eta>0$, there exists a measurable subset $C$ of $A$ and an index $n^{*}$ such that $m(A-C)<2^{-1} \eta$ and $F_{n}$ is finite on $O$ for each $n>n^{*}$. Thus. we may apply Egoroff's theorem to the subsequence $\left\{F_{n^{*}+k}\right\}(k=1,2, \ldots)$ and assert that there exists a subset $B$ of $C$ such that $m(C-B)<2^{-1} \eta$ and $\left\{\boldsymbol{F}_{n^{*}+k}\right\}$ converges uniformly on $B$ to $G$. Consequently, letting $p=n^{*}+k$, for each $\delta>0$, there exists an integer $N_{1}$ such that

$$
\left|F_{p}(x)-G(x)\right|<\delta \text { for each } \quad x \in B \text { and } p>N_{1} .
$$

According to the definition of $F_{p}, F_{p}(x) \geqslant F(x, t)$ for each $x \in A$ and $t \in U_{p}$. Therefore, if $p>N_{1}$,

$$
F^{\prime}(x, t)-\delta<G(x) \quad \text { for each } \quad x \in B \text { and } t \in U_{p} .
$$


According to our hypothesis there exists a neighborhood $V$ of $a$ for which $G(x) \leqslant F^{\prime}(x, t)$ for each $x \in A$ and $t \in \nabla$. Let $N_{2}$ be an integer such that $p>N_{2}$ implies $U_{p} \subset \mathrm{F}$. Then, if $p>N_{2}$,

$$
G(x)<H^{\prime}(x, t)+\delta \quad \text { for each } \quad x \in A \text { and } t \in U_{p} .
$$

Hence if $p>\max \left(N_{1}, N_{2}\right)$ we have $|F(x, t)-G(x)|<\delta$ for each $t \in U_{p}$ and $x \in B$, i.e. $E^{\prime}(\cdot, t)$ converges uniformly to $G$ on $B$ at $a$. Since $m(A-B)$ $<\eta$, our proof is complete.

III. In this section we assume $M C Y$ where $Y$ is a space which is a complete ehain relative to a partial order relation $R$. Then every two elements of $Y$ are $R$-comparable and we may say that $F(x, \cdot)$ is isotone (antitone) on $M$ for each $x \in A$ if $a, b \in M$, aRb imply that $F^{\prime}(x, a) \leqslant F^{\prime}(x, b) \quad\left(F^{\prime}(x, a) \geqslant F(x, b)\right)$. The space $\bar{Y}$ can then be considered a topological space by taking as a basis the "intervals" induced by the partial order $R$. It is known [1] this space is Hausdorff. We assume that $M$ and $Y$ satisfy the further topological conditions stated at the beginning of the second section. The proof of the following theorem is quite similar to that of Theorem 1 and therefore we omit it.

THEOREM 2. If (i) $F(\cdot, t)$ is measurable on $A$ for every $t \in M$, (ii) $F(x, \cdot)$ is monotonic on $M$ for every $x \in A$ and (iii) $\lim _{t \rightarrow a} F(x, t)=G(x)$, a.e. on $A$, then, for each $\eta>0$, there exists a subset $B \subset A$ such that $m(A-B)<\eta$ and the convergence of $F(\cdot, t)$ to $G$ is uniform on $B$.

IV. Although the conditions imposed on $F^{\prime}(x, \cdot)$ in Theorem 1 are stronger than strict semi-continuity, nevertheless, they do not imply continuity of $F^{\prime}(x, \cdot)$ on $M$. Aside from the topologieal conditions imposed on $M$ and $Y$, the question arises whether or not this theorem is really an extension of the result of Hahn and Rosenthal. To see that the conditions imposed in Theorem 1 are significant, we cite the counterexample given by Vinti [9]. Let $M=A=[0,1], a=0$, and let $\left\{\mathbb{E}_{n}\right\}$ be a sequence of mutually disjoint non-measurable sets whose union is $[0,1]$ and such that any finite union of these sets has inner Lebesgue measure zero. Define $\boldsymbol{F}$ as follows:

$$
F(x, t)= \begin{cases}1 & \text { if } \quad x \in E_{n}, t=x / n, x \neq 0, \\ 0 & \text { otherwise. }\end{cases}
$$

Then $\lim _{t \rightarrow 0} F^{\prime}(x, t)=0$ for each $x \in[0,1]$ and $F(\cdot, t)$ is measurable for every $t \epsilon[0,1]$. But $F(\cdot, t)$ does not converge uniformly on any subset of $[0,1]$ of positive measure. We note that $F(x, \cdot)$ is u.s.c. on $[0,1]$ but no neighborhood of $a=0$ exists for which $F(x, t) \leqslant 0$ for each $x \in[0,1]$ and every $t$ in this neighborhood.

\section{References}

[1] G. Birkhoff, Lattice theory, Amer. Math. Soc. Coll. Publ. XXV (1948). [2] N. Bourbaki, Éléments de Mathématique. Livre VI. Intégration, Hermann, Act. Scie. et Ind. 1175, Paris, 1952.

[3] H. Hahn and A. Rosenthal, Set functions, New Mexico, 1948.

[4] D. Hall and G. Spencer, Elementary topology, New York, 1955.

[5] H. Rademacher, Ueber partielle und totale Differenzierbarkeit von Funktionen mehrerer Variablen und ueber die Transformation der Doppelintegrale I, Math. Ann. 79 (1919), pp. 340-359.

[6] S. Saks, Theory of the integral, Stechert and Co., New York, 1937.

[7] W. Stepanoff, Ueber totale Differenzierbarkeit, Math. Ann. 91 (1931), pp. $318-320$.

[8] G. Tolstov, Une remarque sur le théorème de D. F. Egorov, Comptes Rendus Ac. Sciences U.R.S.S., N.S. 22 (1939), pp. 305-307.

[9] C. Vin ti, Una ripartizione del continuou ed una osservazione sulle funzioni continue rispetto ad una e non misurabili rispetto ad un'altra variabile, Rend. Sem. Mat. Univ. Padova 27 (1957), pp. 253-266.

[10] J. D. Weston, $A$ counter-example concerning Egoroff's theorem, J. London Math. Soc. 34 (1959), pp. 139-140.

Reçu par la Rédaction le 31. 3. 1964 